\title{
Airway plumbing
}

John W. Hanrahan

Department of Physiology and Meakins-Christie Laboratories, McGill University, 3655 Drummond Street, Montréal, Québec, Canada H3G 1Y6. Phone: (514) 398-8320; Fax: (514) 398-7452; E-mail: hanrahan@med.mcgill.ca.

Airway surface liquid (ASL), the thin film of fluid covering the luminal aspect of airway epithelial cells, plays a central but mysterious role in cystic fibrosis (CF). CF is characterized by abnormal transepithelial salt transport, viscous airway mucus, chronic bacterial infections, and inflammation, but precisely how mutations in the cystic fibrosis transmembrane conductance regulator (CFTR) gene lead to these symptoms is the subject of a vigorous debate. As discussed one year ago in the Perspective by J.J. Wine (1), there are two very different hypotheses to explain CF pathogenesis in the airways. According to the "volume" model, lack of CFTR in the apical membrane leads to increased salt and fluid absorption by airway epithelial cells, which reduces ASL volume and leads to ineffective mucociliary clearance of bacteria. According to this hypothesis, sodium transport is increased in $\mathrm{CF}$ through upregulation of sodium channels that would normally be inhibited by CFTR (2). The other hypothesis, which we shall refer to here as the "salt" model, proposes that a lack of functional CFTR chloride channels leads to decreased salt absorption, which elevates salt concentration in the ASL, thereby inhibiting activity of antibacterial substances (3). Both hypotheses are strongly supported by in vitro experiments, despite making different predictions concerning the volume and composition of ASL. Fluid collected from the surface of CF airway cell cultures by one group had elevated salt and diminished ability to kill bacteria (3), whereas liquid collected from cultures by the other group was isotonic (2). Different methodologies were used for sampling and analysis, but the contrasting results may simply reflect phenotypic differences between cell cultures prepared in different laboratories. One would like to use the composition of ASL in vivo as the gold standard, but problems associated with sampling a compartment that is only tens of microns thick are even worse in vivo, and those data are also contradictory.
The variable results obtained in vivo may be due to mechanical stimulation during sampling, since this could induce secretion by submucosal glands and change the local ASL volume and composition just as one tries to collect it. Filter paper, which has been widely used to collect ASL, exerts a capillary pressure of about $9 \mathrm{~cm} \mathrm{H}_{2} \mathrm{O}$ (4), which may pull macromolecules and isotonic fluid through the epithelium (5). Most studies indicate that the salt concentration of ASL is somewhat lower than that of plasma, but there is not yet a consensus. For technical reasons, most detailed studies of ASL composition and volume will probably be performed in vitro, at least in the immediate future, which makes validation of cell culture models crucial.

So far, most attention has been focused on salt transport in these models, rather than apical membrane and transepithelial water permeabilities or evaporative loss. The novel confocal microscopy experiments reported by Matsui et al. in this issue of the JCI (6) show that cultured human bronchial epithelial cells have moderately high osmotic water permeability $\left(\mathrm{P}_{\mathrm{f}}\right)$. The value for transepithelial $\mathrm{P}_{\mathrm{f}}$ is consistent with previous reports for freshly excised bovine (7) and porcine (8) trachea and guinea pig distal airways (9). The apical membrane $P_{f}$ of cells grown at the air-liquid interface are slightly higher than those obtained previously for normal and $\mathrm{CF}$ tracheal epithelia cultured under conventional, submerged conditions (10). The moderately high $\mathrm{P}_{\mathrm{f}}$ measured by Matsui et al. (6) favors the volume hypothesis since high $\mathrm{P}_{\mathrm{f}}$, which is characteristic of epithelia such as renal proximal tubule and gall bladder that carry out isosmotic water transport (11), would collapse transepithelial solute gradients. However, to disprove the salt model one needs to show that airway $P_{f}$ is too high to be compatible with the salt hypothesis, and this discriminating value is not known. Presumably $\mathrm{P}_{\mathrm{f}}$ could be somewhat higher than in the renal collecting duct, which, during water diuresis, maintains a much larger salt gradient than the one reported for airway epithelia (12). Regardless, demonstrating that transepithelial $\mathrm{P}_{\mathrm{f}}$ can be studied conveniently in cultured airway epithelia is important and provides hope that conflicting in vitro results will be reconciled. Water and ion permeabilities need to be compared among different in vitro preparations and also with those of native tissue to allow a full understanding of the regulation of ASL salinity and volume. It may be significant that cultures producing hypotonic surface liquid (3) had higher electrical resistance compared with those from which isotonic fluid was collected (2).

The results of Matsui et al. (6) raise interesting questions regarding the molecular basis of $\mathrm{P}_{\mathrm{f}}$ in the bronchial epithelium. In the rat, the bronchial surface epithelium expresses aquaporin-1 (AQP1) in both apical and basolateral membranes (13). The basolateral membrane also contains the mercurial-insensitive water channel AQP4 (14). If AQP4 is the dominant pathway at the basolateral membrane, it may constitute a rate-limiting step for transcellular water flow, since Matsui et al. find that basolateral membrane $\mathrm{P}_{\mathrm{f}}$ is relatively low (6). This could explain the weak sensitivity to mercurials of transepithelial $\mathrm{P}_{\mathrm{f}}$ in cultured bronchial cells and freshly isolated hamster airways (9). On the other hand, the relatively large, mercurial-insensitive component of $\mathrm{P}_{\mathrm{f}}$ at the apical membrane is surprising and hints that AQP4, AQP5, or perhaps a novel apical pathway may exist there in parallel with AQP1.

High apical membrane water permeability would enable water to leave the cells when the tonicity of ASL increases through evaporation (15). Thus, hyperventilation or breathing dry air would elevate ASL osmolality and reduce epithelial cell volume by drawing water from the cells faster than it enters through the basolateral membrane. Matsui et al. make the intriguing suggestion that cell shrinkage could play a sensory role in signaling hypertonicity of the ASL to the microvasculature below (6). Indeed, it was shown years 


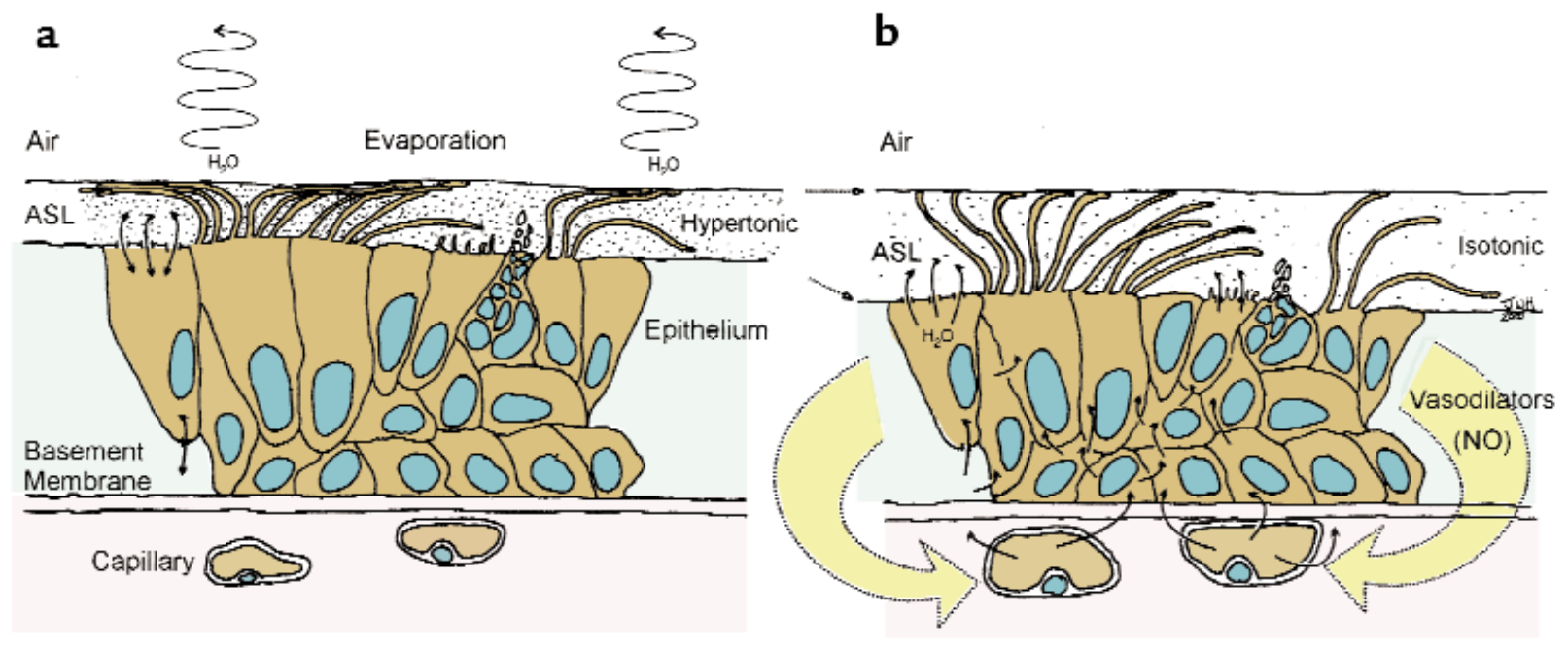

Figure 1

Proposed mechanism for feedback regulation of ASL volume. (a) Evaporation reduces ASL volume, reduces the efficiency of mucociliary clearance, and increases ASL osmolality. (b) High osmotic permeability of the apical membrane allows water to be drawn into the ASL to restore osmolality and causes the cells to shrink. Shrinkage of surface columnar cells triggers release of vasodilator substances such as nitric oxide (NO), which increase fluid delivery by subepithelial microvasculature.

ago that blood flow to the airway submucosa is stimulated by breathing dry air (16) and by hypertonic luminal fluid $(17,18)$. Moreover, several studies (19, 20) have shown that airway epithelia can release smooth muscle-relaxing factors such as nitric oxide (21), and perhaps other vasoactive agents when their luminal surfaces are exposed to hypertonic solution (Figure 1). Although still speculative, this intriguing feedback loop makes sense physiologically, since increasing epithelial perfusion would allow more efficient

1. Wine, J.J. 1999. The genesis of cystic fibrosis lung disease. J. Clin. Invest. 103:309-312.

2. Matsui, H., et al. 1998. Evidence for periciliary liquid layer depletion, not abnormal ion composition, in the pathogenesis of cystic fibrosis airways disease. Cell. 95:1005-1015.

3. Smith, J.J., Travis, S.M., Greenberg, E.P., and Welsh, M.J. 1996. Cystic fibrosis airway epithelia fail to kill bacteria because of abnormal airway surface fluid. Cell. 85:229-236.

4. Landry, J.S., Cowley, E.A., and Eidelman, D.H. 1999. Techniques for ASL collection: role of capillarity. Am. J. Respir. Crit. Care Med. 159:A678. (Abstr.)

5. Erjefält, I., and Persson, C.G.A. 1990. On the use of absorbing discs to sample mucosal surface liquids. Clin. Exp. Allergy. 20:193-197.

6. Matsui, H., Davis, C.W., Tarran, R., and Boucher, R.C. 2000. Osmotic water permeabilities of cultered, well-differentiated normal and cystic fibrosis airway epithelia. J. Clin. Invest. 105:1419-1427.

7. Durand, J., Durand-Arczynska, W., and Haab, P. 1981. Volume flow, hydraulic conductivity and electrical properties across bovine tracheal epithelium in vitro: effect of histamine. Pflügers Arch. 392:40-45.

8. Ballard, S.T., Crews, A.D., and Taylor, A.E. 1998. Hydraulic conductivity of porcine tracheal epithe- rehydration of the ASL.

Understanding the role of ASL in normal and CF airways will require detailed (and preferably simultaneous) studies of solute and water flows under well controlled conditions, which could be varied to probe the effects of hormones and inflammatory mediators from the CF lung on $\mathrm{P}_{\mathrm{f}}$ and $\mathrm{AQP}$ expression. Comparing cultures prepared from normal and aquaporin knockout mice might provide insight into the relative contributions of AQP1, 4 , and 5 to osmotic permeabili-

lium. Am.J. Respir. Crit. Care Med. 157:A556. (Abstr.) 9. Folkesson, H.G., Matthay, M.A., Frigeri, A., and Verkman, A.S. 1996. Transepithelial water permeability in microperfused distal airways. J. Clin. Invest. 97:664-671.

10. Farinas, J., Kneen, M., Moore, M., and Verkman, A.S. 1997. Plasma membrane water permeability of cultured cells and epithelia measured by light microscopy with spatial filtering. J. Gen. Physiol. 110:283-296.

11. Spring, K.R. 1998. Routes and mechanism of fluid transport by epithelia. Annu. Rev. Pbysiol. 60:105-119.

12. Zabner, J., Smith, J.J., Karp, P.H., Widdicomb, J.H., and Welsh, M.J. 1998. Loss of CFTR chloride channels alters salt absorption by cystic fibrosis airway epithelia in vitro. Mol. Cell. 2:397-403.

13. Nielsen, S., King, L.S., Christensen, B., and Agre, P. 1997. Aquaporins in complex tissues. II. Subcellular distribution in respiratory and glandular tissues of rat. Am. J. Physiol. 273:C1549-C1561.

14. Frigeri, A., Gropper, M.A., Turck, C.W., and Verkman, A.S. 1995. Immunolocalization of the mercurial-insensitive water channel and glycerol intrinsic protein in epithelial cell plasma membranes. Proc. Natl. Acad. Sci. USA. 92:4328-4331.

15. Boucher, R.C., Stutts, M.J., Bromberg, P.A., and ty in this region of the airways. The absence of submucosal glands and perhaps other important features of the native epithelium in these culture systems remains an important limitation of such studies, and improved methods are still needed for sampling ASL without affecting the volume or ionic composition of this fluid. Nevertheless, the approach described by Matsui et al. (6) should provide a wealth of new and practical information on epithelial hydrokinetics relevant to cystic fibrosis.

Gatzy, J.T. 1981. Regional differences in airway surface liquid composition. J. Appl. Physiol. 50:613-620.

16. Baile, E.M., Guillemi, S., and Paré, P.D. 1987. Tracheobronchial and upper airway blood flow in dogs during thermally induced panting. J. Appl. Physiol. 63:2240-2246.

17. Deffenbach, M.E., Salonen, R.O., Webber, S.E., and Widdicombe, J.G. 1989. Cold and hyperosmolar fluids in canine trachea: vascular and smooth muscle tone and albumin flux. J. Appl. Physiol. 66:1309-1315.

18. Wells, U.M., Hanafi, Z., and Widdicombe, J.G. 1994. Osmolality alters tracheal blood flow and tracer uptake in anesthetized sheep. J. Appl. Physiol. 77:2400-2407.

19. Hay, D.W.P., Muccitelli, R.M., Horstmeyer, D.L., Wilson, K.A., and Raeburn, D. 1987. Demonstration of the release of an epithelium-derived inhibitory factor from a novel preparation of guinea-pig trachea. Eur. J. Pharmacol. 136:247-259.

20. Munakata, M., Mitzner, W., and Menkes, H. 1988. Osmotic stimuli induce epithelial-dependent relaxation in the guinea pig trachea. J. Appl. Physiol. 64:466-471.

21. Smith, T.L., Prazma, J., Coleman, C.C., Drake, A.F., and Boucher, R.C. 1993. Control of the mucosal microcirculation in the upper respiratory tract. Otolaryngol. Head Neck Surg. 109:646-652. 